\title{
Erratum to: A survey of best practices for RNA-seq data analysis
}

\author{
Ana Conesa ${ }^{1,2^{*}}$, Pedro Madrigal ${ }^{3,4^{*}}$, Sonia Tarazona ${ }^{2,5}$, David Gomez-Cabrero ${ }^{6,7,8,9}$, Alejandra Cervera $^{10}$, \\ Andrew McPherson ${ }^{11}$, Michal Wojciech Szcześniak ${ }^{12}$, Daniel J. Gaffney ${ }^{3}$, Laura L. Elo ${ }^{13}$, Xuegong Zhang ${ }^{14,15}$ \\ and Ali Mortazavi ${ }^{16,17^{*}}$
}

\section{Erratum}

During editing of the article by Conesa et al. [1], an error was introduced to some of the citations, such that incorrect references were provided for some articles the second time they were cited. The following sentences are affected:

Algorithms that quantify expression from transcriptome mappings include RSEM (RNA-Seq by Expectation Maximization) [40], eXpress [41], Sailfish [35] and kallisto [42] among others. These methods allocate multi-mapping reads among transcript and output within-sample normalized values corrected for sequencing biases [35, 41, 43].

The citation for Sailfish should be [34] (Patro et al., Nat Biotechnol. 2014;32:463-4) in both sentences.

Additional factors that interfere with intra-sample comparisons include changes in transcript length across samples or conditions [50], positional biases in coverage along the transcript (which are accounted for in Cufflinks), average fragment size [43], and the GC contents of genes (corrected in the EDAseq package [21]).

The citation for EDAseq should be [20] (Risso et al. BMC Bioinformatics. 2011;12:480)

The NOISeq $R$ package [20] contains a wide variety of diagnostic plots to identify sources of biases in RNA-seq data and to apply appropriate normalization procedures in each case.

The citation for NOISeq should be [19] (Tarazona et al. Nucleic Acids Res. 2015;43:e140)

These effects can be minimized by appropriate experimental design [51] or, alternatively, removed by batch-correction methods such as COMBAT [52] or ARSYN [20, 53].

\footnotetext{
*Correspondence: aconesa@ufl.edu; pm12@sanger.ac.uk; ali.mortazavi@uci.edu 'Institute for Food and Agricultural Sciences, Department of Microbiology and Cell Science, University of Florida, Gainesville, FL 32603, USA

${ }^{3}$ Wellcome Trust Sanger Institute, Wellcome Trust Genome Campus, Hinxton, Cambridge CB10 1SA, UK

${ }^{16}$ Department of Developmental and Cell Biology, University of California, Irvine, Irvine, CA 92697-2300, USA

Full list of author information is available at the end of the article
}

The citations for ARSyN should be [19,53] (Tarazona et al. Nucleic Acids Res. 2015;43:e140, Nueda et al. Biostatistics. 2012;13:553-66).

All these approaches are generally hampered by the intrinsic limitations of short-read sequencing for accurate identification at the isoform level, as discussed in the RNA-seq Genome Annotation Assessment Project paper [30].

The citation for the RGASP article should be [29] (Engström et al. Nat Methods. 2013;10:1185-91).

We refer the reader to [30] for a comprehensive comparison of RNA-seq mappers.

This citation should be [29] (Engström et al. Nat Methods. 2013;10:1185-91).

\section{Author details}

'Institute for Food and Agricultural Sciences, Department of Microbiology and Cell Science, University of Florida, Gainesville, FL 32603, USA. ${ }^{2}$ Centro de Investigación Príncipe Felipe, Genomics of Gene Expression Laboratory, 46012 Valencia, Spain. ${ }^{3}$ Wellcome Trust Sanger Institute, Wellcome Trust Genome Campus, Hinxton, Cambridge CB10 1SA, UK. "Wellcome

Trust-Medical Research Council Cambridge Stem Cell Institute, Anne McLaren Laboratory for Regenerative Medicine, Department of Surgery, University of Cambridge, Cambridge CB2 0SZ, UK. ${ }^{5}$ Department of Applied Statistics, Operations Research and Quality, Universidad Politécnica de Valencia, 46020, Valencia, Spain. ${ }^{6}$ Unit of Computational Medicine, Department of Medicine, Karolinska Institutet, Karolinska University Hospital, 17177 Stockholm, Sweden. ${ }^{7}$ Center for Molecular Medicine, Karolinska Institutet, 17177 Stockholm, Sweden. ${ }^{8}$ Unit of Clinical Epidemiology, Department of Medicine, Karolinska University Hospital, L8, 17176 Stockholm, Sweden. ${ }^{9}$ Science for Life Laboratory, 17121 Solna, Sweden. ${ }^{10}$ Systems Biology Laboratory, Institute of Biomedicine and Genome-Scale Biology Research Program, University of Helsinki, 00014 Helsinki, Finland. ${ }^{11}$ School of Computing Science, Simon Fraser University, Burnaby V5A 1S6BC, Canada. ${ }^{12}$ Department of Bioinformatics, Institute of Molecular Biology and Biotechnology, Adam Mickiewicz University in Poznań, 61-614 Poznań, Poland. ${ }^{13}$ Turku Centre for Biotechnology, University of Turku and Åbo Akademi University, Fl-20520 Turku, Finland. ${ }^{14}$ Key Lab of Bioinformatics/Bioinformatics Division, TNLIST and Department of Automation, Tsinghua University, Beijing 100084, China. ${ }^{15}$ School of Life Sciences, Tsinghua University, Beijing 100084, China. ${ }^{16}$ Department of Developmental and Cell Biology, University of California, Irvine, Irvine, CA 92697-2300, USA. ${ }^{17}$ Center for Complex Biological Systems, University of California, Irvine, Irvine, CA 92697, USA.

Received: 17 August 2016 Accepted: 17 August 2016 Published online: 26 August 2016 


\section{References}

1. Conesa A, Madrigal P, Tarazona S, Gomez-Cabrero D, Cervera A, McPherson A, et al. Genome Biol. 2016;17:13.

Submit your next manuscript to BioMed Central and we will help you at every step:

- We accept pre-submission inquiries

- Our selector tool helps you to find the most relevant journal

- We provide round the clock customer support

- Convenient online submission

- Thorough peer review

- Inclusion in PubMed and all major indexing services

- Maximum visibility for your research

Submit your manuscript at www.biomedcentral.com/submit 